\title{
Monocyte Gene Expression Signatures in Rheumatic Diseases: Biomarkers for Disease Activity and Tools for Diagnosis and Classification
}

\author{
Zana Brkic, Evelyn D. Olthof, Hemmo A. Drexhage and Marjan A. Versnel*
}

Department of Immunology, Erasmus MC University Medical Center, Rotterdam, The Netherlands

\begin{abstract}
Monocytes migrate through the blood to the peripheral tissues, where they can develop into dendritic cells (DC) and macrophages. Studies on the phenotype of peripheral blood monocytes in rheumatic diseases revealed changes in the number of CD16+ monocytes and in the adhesive, chemotactic, antigen presenting and inflammatory properties of the cells. However, these studies often resulted in fragmented and inconsistent data and are hampered by the heterogeneity and overlap of the rheumatic diseases.

By microarray analysis, changes in gene expression-profiles are detectable and subsequent correlation of the data reveals functional pathways forming a "gene expression signature". Determination of monocyte gene expression signatures in patient groups with rheumatic diseases has resulted in the detection of an Interferon (IFN) Type I induced signature in subgroups of patients with rheumatoid arthritis, Sjögren's Syndrome, systemic sclerosis and systemic lupus erythematosus. We assume that such profiling, which is robust, will lead to the development of better classification criteria and an insight into at least one functional pathogenic pathway leading to disease, i.e. the one mediated by IFN type 1.
\end{abstract}

Keywords: Monocytes, IFN type I, Rheumatic diseases, Sjogren's Syndrome.

\section{MONOCYTES AND THE MONONUCLEAR PHAGO- CYTE SYSTEM (MPS)}

Monocytes are part of the Mononuclear Phagocyte System (MPS) system. Cells of the MPS system are thought to originate from pro-monocytes in the bone marrow and to migrate as monocytes through the blood to the peripheral tissues, where they replenish the pool of tissue and inflammatory macrophages. In addition, monocytes can function as precursors of the so-called monocyte-derived dendritic cells (DC). DC and macrophages are present in virtually all tissues and play an important role in tissue homeostasis (growth and function of parenchymal cells), the removal of debris and the regulation of the immune response. In addition to the blood monocyte as a precursor for tissue macrophages and DC, evidence is accumulating that in many tissues monocyte-like precursors for macrophages and DC are present locally. Under steady state conditions, DC and tissue macrophages are in a tolerogenic state, interacting with $\mathrm{T}$ cells in such a way that the induction of autoimmune responses is prevented by a preferential generation of tolerogenic regulator $\mathrm{T}$ cells. Under inflammatory conditions $\mathrm{DC}$ and macrophages can initiate effector $\mathrm{T}$ cells responses resulting in the induction of specific immunity and the ultimate elimination of pathogens. Monocytes can be divided into different subsets according to phenotype and function [1]. In humans, two populations have been distinguished based upon the expression of CD14 and CD16. The majority of monocytes is CD14+ and CD16-, while approximately $10 \%$ of the monocytes is CD14+ and CD16+. Further phenotypic characterization of the monocyte subsets revealed that the

*Address correspondence to this author at the Department of Immunology, Erasmus MC, Rotterdam, The Netherlands; Tel: 0031107044086; Fax: 0031107044731; E-mail: m.versnel@erasmusmc.nl
CD16+ monocytes have a high expression of the CX3CR1 receptor and low levels of CCR2 and CD62L [2]. This latter population is considered to be relatively mature in comparison to CD14+CD16- monocytes [3,4]. The immature CD16population is considered to be the population from which the inflammatory monocytes are recruited during inflammation, while the mature population would be precursors of the steady state tissue macrophages and DC. Recently, it has been proposed that the $\mathrm{CD} 16+$ monocyte subset can be further sub-divided into two dinstinct functional subsets, CD14 (high)CD16+ and CD14(dim)CD16+ [5,6]. Future studies will no doubt reveal the presence of more functionally distinct subsets and their contribution to inflammation and autoimmunity.

In summary, the family of monocytes, macrophages and DC is a large family of functionally and developmentally related cells that are essential for the induction of tolerance towards autoantigens (non-dangerous steady state conditions) or the building of effective immune responses to dangerous intruders. Peripheral blood monocytes can act as precursors for DC and macrophages and offer an opportunity to study the MPS system in autoimmune diseases and identify abnormalities that contribute to the failure of the immune system to preserve a tolerogenic steady state.

\section{ABBERATIONS OF MONOCYTES IN RHEUMATIC DISEASES}

Animal models for autoimmunity allow the study of the earliest steps in the development of the disease. These studies revealed macrophages and DC to be the first cells accumulating in the target organ, e.g. the synovium in rheumatoid arthritis (RA), the islets in Type 1 diabetes (T1D) and the salivary glands in Sjögren's Syndrome (SjS) [7-10]. During the development of such autoimmune diseases the accumula- 
tion of monocytes and monocyte-derived cells in target organs is crucial for the induction and perpetuation of the autoimmune response.

In particular we have studied the development of $\mathrm{SjS}$ and T1D in the Non-Obese Diabetic (NOD) mouse. In the preautoimmune animal (3-7 weeks of age) an accumulation of DC occurred in the salivary glands and pancreas before the start of any noteworthy $\mathrm{T}$ cell infiltration. This observation underscores the important role of DC in the pathogenesis of the disease. We hypothesized that intrinsic abnormalities in dendritic and related cells contribute to the abnormal accumulation of DC in the target organs. Indeed several investigators have reported on an abnormal functioning and trafficking of DC and macrophages in the NOD mouse prior to the outbreak of the autoimmune disease [11-16], suggesting an incapability of the cells to induce tolerance towards autoantigens. Correction of the aberrant function of DC via introduction of corrected DC has been shown to lead to the prevention and/or delay of onset of autoimmunity in the NOD mouse [17, 18].

Considering the important role of DC and macrophages in the initiation of autoimmunity and that peripheral blood monocytes are important precursors for DC and macrophages, peripheral blood monocytes of patients with autoimmune diseases were studied for phenotypic and functional abnormalities.

In monocytes of T1D patients a raised production of interleukin (IL)-1 $\beta$, IL-6, superoxide anion, and prostaglandinendoperoxide synthase 2 (PTGS2) [19-21], an aberrant generation of antigen-presenting cells [22, 23] and abnormal chemotaxis, adhesion, and migratory potential [24] have been described.

With regard to rheumatic diseases, studies on the phenotype of peripheral blood monocytes revealed several abnormalities. An increase in the CD14+CD16+ subset was observed in RA and systemic lupus erythematosus (SLE) patients [25-27, unpublished results]. However for RA a decrease of this population has also been reported [28]. We recently described a significant increase in the CD14+CD16+ subset in primary SjS (pSjS) [27]. These mature CD14+CD16+ monocytes displayed a normal expression of chemokine receptors and adhesion molecules. Using an in vitro reverse transmigration assay we demonstrated that DC generated from the CD14+CD16+ subset displayed a similar phenotype as the $\mathrm{DC}$ in $\mathrm{pSjS}$ salivary glands. These data support a role for CD14+CD16+ monocytes in the pathogenesis of $\mathrm{pSjS}$.

In addition to shifts in monocyte subpopulations other aberrancies in monocytes in rheumatic diseases have been reported. In RA an increased expression of Fc receptors and an increased adhesion to the extracellular matrix has been described [29, 30]. In addition, an activated population of CCR5+ monocytes was found in the synovial fluid of RA patients indicative for a role of these cells in the pathogenesis of RA [31, 32]. The relationship between disease activity and an enhanced expression of the Dipeptidyl Peptidase IV density on monocytes of RA patients also underscores the contribution of abnormally functioning monocytes to the disease development [33].
Blocking TNF-alpha has a beneficial effect in a proportion of RA patients. Interestingly, monocytes of RA patients have an increased activation of nuclear factor-kappaB $(\mathrm{NF} \mathrm{B})$ that correlates to peripheral blood TNF-alpha levels [34]. Ex vivo treatment with infliximab and etanercept resulted in decreased activation of $\mathrm{NF \kappa B}$ in monocytes and not lymphocytes, further underscoring the important role of monocytes in the pathogenesis of RA.

In SLE a defective phagocytosis of monocytes and macrophages has been implicated in the pathogenesis of this systemic autoimmune disease. The envisaged cascade of events is thought to be triggered by impaired phagocytosis of monocytes/macrophages, resulting in the accumulation of apoptotic nuclear debris leading to the triggering of autoimmune phenomena towards nuclear antigens, including antinuclear antibodies. Subsequently, immune complexes accumulate resulting in the tissue damage observed in SLE [35]. However such a phagocytic clearance deficiency has only been observed in a subgroup of patients [35]. Recently the theory was expanded to include much broader abnormalities in monocytes and macrophages being involved in the basis of the disease after observations in SLE animal models [36]. Apart from an increased production of inflammatory mediators and increased expression of activation induced molecules by monocytes, some SLE patients exhibit monocytes that function as highly efficient antigen presenting cells in an in vitro mixed lymphocyte reaction [37]. This "DC like" behavior of the monocytes, that is absent in healthy control cells, is probably caused by the presence of IFN alpha in the serum of the patients and might lead to the activation of autoreactive $\mathrm{T}$ cells

Systemic Sclerosis (SSc), is a rheumatic disease characterized by fibrosis, vascular abnormalities and pulmonary hypertension. The etiology and pathogenesis of this disease is poorly understood [38]. In the pathogenesis of this generalized autoimmune disease monocytes are also considered to play an important role by interacting with endothelial cells and fibroblasts via the production of inflammatory factors. Furthermore the so-called circulating fibrocyte precursor, a monocytic cell in the blood, is considered to be a potential precursor for fibroblasts. Interestingly monocytes of early SSc patients (and not of chronic SSc, RA and SLE patients) show an up regulation of the tumor necrosis factor converting enzyme (TACE) [39]. However, further phenotyping of CD14+ monocytes by flowcytometry in SSc patients with limited disease did not reveal differences in a number of the cell surface markers associated with activation, migration and transformation [40].

In summary, the phenotypic and functional analysis of monocytes in rheumatic diseases is elaborate and has revealed a heterogeneous picture with inconsistent and sometimes contradictory results. Functional assays as described above are apparently not precise and robust enough to determine the complex abnormalities of monocytes in rheumatic disease patients. The heterogeneity of rheumatic diseases is another complicating factor.

\section{MONOCYTE GENE EXPRESSION PROFILING: A NOVEL APPROACH}

Our recent studies show that by microarray analysis changes in gene expression profiles can be detected in 
monocytes. A subsequent correlation of the data reveals functional pathways forming a specific "gene expression signature", illustrating an abnormal pattern of monocyte activation (an abnormal "set point"). These monocyte gene expression profiles and set points can be used for the study of large patient groups and the development of better classification criteria. It is important to note that in our experience, the more homogenous the patient groups and cell samples the clearer the results of such analysis.

Our microarray analysis were performed with CD14+ purified monocyte fractions because of the important role of monocytes and monocyte-derived cells in the pathogenesis of autoimmune diseases and the variability of the different cell types present in a PBMC fraction. Our profiling of CD14+ monocytes resulted in the identification of gene expression signatures for $\mathrm{T} 1 \mathrm{D}, \mathrm{pSjS}$ and autoimmune thyroiditis [41, 42, Drexhage et al. unpublished results]. We detected two different signatures in T1D: a signature involving primarily cytokine/inflammatory compound genes and a second signature that involves genes of chemotaxis, adhesion, motility and metabolism [42]. Interestingly, this latter gene expression signature is in line with our earlier observations on functional adhesion and chemotaxis abnormalities of monocytes of T1D patients $[43,44]$ underscoring the usefulness of gene profiling for unraveling functional pathways. Furthermore the existence of two different gene expression signatures can clarify conflicting data reported on the proinflammatory state of monocytes in T1D and the two detected profiles provide a new method of classification of autoimmune diabetes.

For $\mathrm{pSjS}$ we used a similar approach resulting in the detection of an IFN type I gene expression signature in monocytes of patients [41]. Interestingly, the monocyte IFN type I signature is only present in a subgroup of patients with $\mathrm{pSjS}$ and correlates with disease activity (Brkic, unpublished results). Earlier observations on IFN type I activity in patients pointed towards a limited effect in the salivary glands since serum IFN type I could only be detected in a few patients [45-47]. Given a putative role for IFN type I in $\mathrm{pSjS}$ the next logical next step would be to treat these patients with inhibitors of the IFN pathway using an anti-IFN type I monoclonal antibody or antibodies blocking the IFN type I receptor [48].

A subgroup of RA patients also displays the IFN expression signature in their monocytes (Versnel, unpublished results). This observation is consistent with data on PBMC profiling in RA [49]. Profiling of whole blood or peripheral blood mononuclear cells (PBMC) has been performed in rheumatic diseases in the assumption that PBMC gene expression profiles reflect the situation in the target tissues. This approach has also resulted in the identification of the IFN expression signature in peripheral blood mononuclear cells (PBMC) of patients with SLE, RA and SSc [49-51], despite the use of non- purified PBMC suspensions. For SLE the contribution of IFN type I in the pathogenesis of the disease had earlier been established using a variety of other approaches [48,52]. Although the use of PBMC thus suffices for the detection the IFN gene expression signature in rheumatic diseases, we favor the use of purified CD14+ monocytes. The importance of the use of purified monocytes is supported by data from Batliwalla et al. [53] which shows that differences in monocyte counts between healthy controls and patients with RA influence the results obtained when profiling PBMC. Actually, most genes identified in this study were genes activated in monocytes. Moreover, in T1D it is essential to use monocytes, since the other cell populations contaminate and obscure the specific abnormal gene expression patterns found in the T1D monocytes.

The present concept on IFN type I in rheumatic diseases is that plasmacytoid DC secrete IFN type I in response to activation by e.g. immune complexes containing nucleic acids. Subsequently, this IFN type I activity results in an up regulation of a large number of IFN type 1 induced genes. In particular, the effect of IFN type I on monocytes seems to be important for the pathogenesis of rheumatic diseases since IFN type I induces monocytes to differentiate into DC and to express B cell-activating factor (BAFF). BAFF in turn contributes to the survival and hyperactivity of autoreactive $B$ cells, a hallmark of various rheumatic diseases [54].

\section{CONCLUSION AND FUTURE DIRECTIONS}

Rheumatic diseases are presently diagnosed by a combination of different tests that lack specificity for a single disease and vary within a disease group. Treatment of rheumatic diseases is frequently non-specific and not effective in all patients. Furthermore, the chronic nature with remissions and relapses in rheumatic diseases urges the identification of reliable biomarkers for disease activity.

Identification of monocyte gene expression signatures in larger patient samples will provide insight into the pathogenesis of rheumatic diseases and provide tools for new classification methods. As the identified monocyte signatures are based on functional pathways involved in these diseases, new options for specific treatment of the various subgroups will become feasible. Furthermore, any such specific treatment could possibly be monitored by using the monocyte signatures. We predict that in the future an accurate monocyte profiling will precede and determine the treatment of a patient with a rheumatic disease.

\section{REFERENCES}

[1] Geissmann F, Auffray C, Palframan R, et al. Blood monocytes: distinct subsets, how they relate to dendritic cells, and their possible roles in the regulation of T-cell responses. Immunol Cell Biol 2008; 86: 398-408

[2] Ancuta P, Rao R, Moses A, et al. Fractalkine preferentially mediates arrest and migration of CD16+ monocytes. J Exp Med 2003; 97: 1701-7.

[3] Ziegler-Heitbrock HW, Fingerle G, Ströbel M, et al. The novel subset of CD14+/CD16+ blood monocytes exhibits features of tissue macrophages. Eur J Immunol 1993; 23: 2053-8.

[4] Ziegler-Heitbrock L. The CD14+ CD16+ blood monocytes: their role in infection and inflammation. J Leukoc Biol 2007; 81: 58492.

[5] Auffray C, Sieweke MH, Geissmann F. Blood monocytes: development, heterogeneity, and relationship with dendritic cells. Annu Rev Immunol 2009; 27: 669-92.

[6] Skrzeczyńska-Moncznik J, Bzowska M, Loseke S, GrageGriebenow E, Zembala M, Pryjma J. Peripheral blood CD14high CD16+ monocytes are main producers of IL-10. Scand J Immunol 2008; 67: 152-9.

[7] Ziegler AG, Erhard J, Lampeter EF, Nagelkerken LM, Standl E. Involvement of dendritic cells in early insulitis of BB rats. J Autoimmun 1992; 5: 571-9.

[8] Jansen A, Homo-Delarche F, Hooijkaas H, Leenen PJ, Dardenne M, Drexhage HA. Immunohistochemical characterization of monocytes-macrophages and dendritic cells involved in the initiation of 
the insulitis and $\beta$-cell destruction in NOD mice. Diabetes 1994; 43: 667-5.

[9] van Blokland SC, van Helden-Meeuwsen CG, Wierenga-Wolf AF, et al. Two different types of sialoadenitis in the NOD- and MRL/lpr mouse models for Sjögren's syndrome: a differential role for dendritic cells in the initiation of sialoadenitis? Lab Invest 2000; 80: 575-85.

[10] Thomas R, Davis LS, Lipsky PE. Rheumatoid synovium is enriched in mature antigen-presenting dendritic cells. J Immunol 1994; 152: 2613-23

[11] Sen P, Bhattacharyya S, Wallet M, et al. NF-kappa B hyperactivation has differential effects on the APC function of nonobese diabetic mouse macrophages. J Immunol 2003; 170: 1770-80.

[12] Nikolic T, Bunk M, Drexhage HA, Leenen PJ. Bone marrow precursors of nonobese diabetic mice develop into defective macrophage-like dendritic cells in vitro. J Immunol 2004; 173: 4342-51.

[13] Sommandas V, Rutledge EA, Van Yserloo B, Fuller J, Lernmark A, Drexhage HA. Aberrancies in the differentiation and maturation of dendritic cells from bone-marrow precursors are linked to various genes on chromosome 4 and other chromosomes of the BB-DP rat. J Autoimmun 2005; 25: 1-12.

[14] Bouma G, Nikolic T, Coppens JM, et al. NOD mice have a severely impaired ability to recruit leukocytes into sites of inflammation. Eur J Immunol 2005; 35: 225-35.

[15] Geutskens SB, Nikolic T, Dardenne M, Leenen PJ, Savino W. Defective up-regulation of CD49d in final maturation of NOD mouse macrophages. Eur J Immunol 2004; 34: 3465-76.

[16] Wildenberg ME, van Helden-Meeuwsen CG, van de Merwe JP, Moreno C, Drexhage HA, Versnel MA. Lack of CCR5 on dendritic cells promotes a proinflammatory environment in submandibular glands of the NOD mouse. J Leukoc Biol 2008; 83: 1194-200.

[17] Feili-Hariri M, Falkner DH, Morel PA. Regulatory Th2 response induced following adoptive transfer of dendritic cells in prediabetic NOD mice. Eur J Immunol 2002; 32: 2021-30.

[18] Adorini L. Tolerogenic dendritic cells induced by vitamin D receptor ligands enhance regulatory $\mathrm{T}$ cells inhibiting autoimmune diabetes. Ann N Y Acad Sci 2003; 987: 258-61.

[19] Plesner A, Greenbaum CJ, Gaur LK, Ernst RK, Lernmark A. Macrophages from high-risk HLA-DQB1*0201/*0302 type 1 diabetes mellitus patients are hypersensitive to lipopolysaccharide stimulation. Scand J Immunol 2002; 56: 522-9.

[20] Devaraj S, Glaser N, Griffen S, Wang-Polagruto J, Miguelino E, Jialal I. Increased monocytic activity and biomarkers of inflammation in patients with type 1 diabetes. Diabetes 2006; 55: 774-9.

[21] Litherland SA, She JX, Schatz D, et al. Aberrant monocyte prostaglandin synthase 2 (PGS2) expression in type 1 diabetes before and after disease onset. Pediatr Diabetes 2003; 4: 10-8.

[22] Skarsvik S, Tiittanen M, Lindstrom A, Casas R, Ludvigsson J, Vaarala O. Poor in vitro maturation and pro-inflammatory cytokine response of dendritic cells in children at genetic risk of type 1 diabetes. Scand J Immunol 2004; 60: 647-52.

[23] Takahashi K, Honeyman MC, Harrison LC. Impaired yield, phenotype, and function of monocyte-derived dendritic cells in humans at risk for insulin-dependent diabetes. J Immunol 1998; 161: 2629-35.

[24] Bouma G, Lam-Tse WK, Wierenga-Wolf AF, Drexhage HA, Versnel MA. Increased serum levels of MRP-8/14 in type 1 diabetes induce an increased expression of CD11b and an enhanced adhesion of circulating monocytes to fibronectin. Diabetes 2004; 53: 1979-86.

[25] Kawanaka N, Yamamura M, Aita T, et al. CD14+,CD16+ blood monocytes and joint inflammation in rheumatoid arthritis. Arthritis Rheum 2002; 46: 2578-86.

[26] Sullivan KE, Suriano A, Dietzmann K, Lin J, Goldman D, Petri MA. The TNFalpha locus is altered in monocytes from patients with systemic lupus erythematosus. Clin Immunol 2007; 123: 7481.

[27] Wildenberg ME, Welzen-Coppens JM, van Helden-Meeuwsen CG, et al. Increased frequency of CD16+ monocytes and the presence of activated dendritic cells in salivary glands in primary Sjögren syndrome. Ann Rheum Dis 2009; 68: 420-6.

[28] Cairns AP, Crockard AD, Bell AL. The CD14+ CD16+ monocyte subset in rheumatoid arthritis and systemic lupus erythematosus. Rheumatol Int 2002; 21: 189-92.

[29] Hepburn AL, Mason JC, Davies KA. Expression of Fcgamma and complement receptors on peripheral blood monocytes in systemic lupus erythematosus and rheumatoid arthritis. Rheumatology 2004; 43: $547-54$.

[30] Wijngaarden S, van de Winkel JG, Jacobs KM, Bijlsma JW, Lafeber FP, van Roon JA. A shift in the balance of inhibitory and activating Fcgamma receptors on monocytes toward the inhibitory Fcgamma receptor $\mathrm{IIb}$ is associated with prevention of monocyte activation in rheumatoid arthritis. Arthritis Rheum 2004; 50: 387887.

[31] Katschke KJ Jr, Rottman JB, Ruth JH, et al. Differential expression of chemokine receptors on peripheral blood, synovial fluid, and synovial tissue monocytes/macrophages in rheumatoid arthritis. Arthritis Rheum 2001; 44: 1022-32.

[32] Kohem CL, Brenol JC, Xavier RM, et al. The chemokine receptor CCR5 genetic polymorphism and expression in rheumatoid arthritis patients. Scand J Rheumatol 2007; 36: 359-64.

[33] Ellingsen T, Hornung N, Møller BK, Hjelm-Poulsen J, StengaardPedersen K. In active chronic rheumatoid arthritis, dipeptidyl peptidase IV density is increased on monocytes and CD4(+) T lymphocytes. Scand J Immunol 2007; 66: 451-7.

[34] Dichamp I, Bourgeois A, Dirand C, Herbein G, Wendling D. Increased nuclear factor-kappaB activation in peripheral blood monocytes of patients with rheumatoid arthritis is mediated primarily by tumor necrosis factor-alpha. J Rheumatol 2007; 34: 1976-83.

[35] Gaipl US, Munoz LE, Grossmayer G, et al. Clearance deficiency and systemic lupus erythematosus (SLE). J Autoimmun 2007; 28 : 114-21.

[36] Katsiari CG, Liossis SN, Sfikakis PP. The pathophysiologic role of monocytes and macrophages in systemic lupus erythematosus: A reappraisal. Semin Arthritis Rheum 2009; [Epub a head of print].

[37] Blanco P, Palucka AK, Gill M, Pascual V, Banchereau J. Induction of dendritic cell differentiation by IFN-alpha in systemic lupus erythematosus. Science 2001; 294: 1540-3.

[38] Tamby MC, Chanseaud Y, Guillevin L, Mouthon L. New insights into the pathogenesis of systemic sclerosis. Autoimmun Rev 2003; 2: $152-7$

[39] Bohgaki T, Amasaki Y, Nishimura N, et al. Up regulated expression of tumour necrosis factor $\{$ alpha $\}$ converting enzyme in peripheral monocytes of patients with early systemic sclerosis. Ann Rheum Dis 2005; 64: 1165-73.

[40] Russo R, Medbury H, Guiffre A, Englert H, Manolios N. Lack of increased expression of cell surface markers for circulating fibrocyte progenitors in limited scleroderma. Clin Rheumatol 2007; 26: 1136-41.

[41] Wildenberg ME, van Helden-Meeuwsen CG, van de Merwe JP, Drexhage HA, Versnel MA. Systemic increase in type I interferon activity in Sjögren's syndrome: a putative role for plasmacytoid dendritic cells. Eur J Immunol 2008; 38: 2024-33.

[42] Padmos RC, Schloot NC, Beyan H, et al. LADA Consortium. Distinct monocyte gene-expression profiles in autoimmune diabetes. Diabetes 2008; 57: 2768-73.

[43] Bouma G, Lam-Tse WK, Wierenga-Wolf AF, Drexhage HA, Versnel MA. Increased serum levels of MRP-8/14 in type 1 diabetes induce an increased expression of CD11b and an enhanced adhesion of circulating monocytes to fibronectin. Diabetes 2004; 53: 1979-86.

[44] Bouma G, Coppens JM, Lam-Tse WK, et al. An increased MRP8/14 expression and adhesion, but a decreased migration towards proinflammatory chemokines of type 1 diabetes monocytes. Clin Exp Immunol 2005; 141: 509-17.

[45] Hjelmervik TO, Petersen K, Jonassen I, Jonsson R, Bolstad AI. Gene expression profiling of minor salivary glands clearly distinguishes primary Sjögren's syndrome patients from healthy control subjects. Arthritis Rheum 2005; 52: 1534-44.

[46] Båve U, Nordmark G, Lövgren T, et al. Activation of the type I interferon system in primary Sjögren's syndrome: a possible etiopathogenic mechanism. Arthritis Rheum 2005; 52: 1185-95.

[47] Gottenberg JE, Cagnard N, Lucchesi C, et al. Activation of IFN pathways and plasmacytoid dendritic cell recruitment in target organs of primary Sjögren's syndrome. Proc Natl Acad Sci USA 2006; 103: 2770-5.

[48] Rönnblom L, Pascual V. The innate immune system in SLE: type I interferons and dendritic cells. Lupus 2008; 17: 394-9.

[49] van der Pouw Kraan TC, Wijbrandts CA, van Baarsen LG, et al. Rheumatoid arthritis subtypes identified by genomic profiling of peripheral blood cells: assignment of a type I interferon signature in a subpopulation of patients. Ann Rheum Dis 2007; 66: 1008-14. 
[50] Baechler EC, Batliwalla FM, Karypis G, et al. Interferon-inducible gene expression signature in peripheral blood cells of patients with severe lupus. Proc Natl Acad Sci USA 2003; 100: 2610-5.

[51] Bos CL, van Baarsen LG, Timmer TC, et al. Molecular subtypes of systemic sclerosis in association with anti-centromere antibodies and digital ulcers. Genes Immun 2009; 10: 210-8.

[52] Pascual V, Allantaz F, Patel P, Palucka AK, Chaussabel D, Banchereau J. How the study of children with rheumatic diseases identified interferon-alpha and interleukin-1 as novel therapeutic targets. Immunol Rev 2008; 223: 39-59.

[53] Batliwalla FM, Baechler EC, Xiao X, et al. Gregersen PK. Peripheral blood gene expression profiling in rheumatoid arthritis. Genes Immun 2005; 6: 388-97.

[54] Litinskiy MB, Nardelli B, Hilbert DM, et al. DCs induce CD40independent immunoglobulin class switching through BLyS and APRIL. Nat Immunol 2002; 3: 822-9.

(C) Brkic et al.; Licensee Bentham Open.

This is an open access article licensed under the terms of the Creative Commons Attribution Non-Commercial License (http://creativecommons.org/licenses/by-nc/3.0/) which permits unrestricted, non-commercial use, distribution and reproduction in any medium, provided the work is properly cited. 